Note

\title{
Structural Analysis of a Novel Oligosaccharide Isolated from Fermented Beverage of Plant Extracts
}

(Received August 16, 2017; Accepted October 17, 2017)

(J-STAGE Advance Published Date: October 27, 2017)

\author{
Akira Yamamori, ${ }^{1, \dagger}$ Yusuke Takata, ${ }^{2}$ Eri Fukushi, ${ }^{2}$ Jun Kawabata, ${ }^{2}$ Hideki Okada,${ }^{1}$ Naoki Kawazoe, ${ }^{1}$ \\ Keiji Ueno, ${ }^{3}$ Shuichi Onodera, ${ }^{3}$ and Norio Shiomi ${ }^{3}$ \\ ${ }^{1}$ General Institute of Ohtakakohso Co., Ltd. \\ (1-22-10 Sakura, Otaru 047-0193, Japan)) \\ ${ }^{2}$ Graduate School of Agriculture, Hokkaido University \\ (Sapporo 060-8589, Japan) \\ ${ }^{3}$ Department of Food and Nutrition Sciences, Graduate School of Dairy Science Research, Rakuno Gakuen University \\ (582-1 Bunkyodai-Midorimachi, Ebetsu 069-8501, Japan)
}

\begin{abstract}
A fermented beverage of plant extracts (Super Ohtaka ${ }^{\circledR}$ ) was prepared from about 50 kinds of fruits and vegetables. This natural fermentation was performed by yeast (Zygosaccharomyces spp. and Pichia spp.) and lactic acid bacteria (Leuconostoc spp.) and resulted in the production of a novel fructopyranose-containing saccharide, which was subsequently isolated using carbon-Celite column chromatography and preparative-HPLC. The structure of the saccharide was determined using MALDI-TOF MS and NMR, and the saccharide was identified as $\beta$-D-fructopyranosyl-(2 $\rightarrow 6)-\beta$-Dfructofuranosyl-(2↔1)- $\alpha$-D-glucopyranoside. This is the first description of this novel saccharide and its isolation from a natural source.
\end{abstract}

Key words: pyrano-6-kestose, fructopyranosyl sucrose, fructopyranose, trisaccharide, fermented beverage, structural analysis

Super Ohtaka ${ }^{\circledR}$ (fermented beverage of plant extracts ${ }^{1)}$ ) displayed scavenging activity against the radical 1,1'-diphenyl-2-picrylhydrazyl and significantly reduced ethanolinduced damage of the gastric mucosa in rats. ${ }^{1)}$ This beverage contained primarily glucose and fructose, but also various oligosaccharides as well. We have previously analyzed the structures of some of these oligosaccharides, including $\beta$-D-fructopyranosyl- $(2 \rightarrow 6)$-D-glucopyranose $\quad(\beta$ Fp $2 \rightarrow 6 \mathrm{G}),{ }^{2}{ }^{2} \quad \alpha$-D-fructofuranosyl-(2 $\left.\rightarrow 6\right)$-D-glucopyranose $(\alpha-\mathrm{F} f 2 \rightarrow 6 \mathrm{G}),{ }^{2)} \beta$-D-fructopyranosyl-( $\left.2 \rightarrow 6\right)-\beta$-D-glucopyranosyl-( $1 \rightarrow 3)$-D-glucopyranose, ${ }^{3)} \quad \beta$-D-fructopyranosyl$(2 \rightarrow 6)$-D-fructofuranose, ${ }^{4} \quad$ and $\quad \beta$-D-fructopyranosyl( $2 \rightarrow 1)-\beta$-D-fructofuranosyl-( $2 \leftrightarrow 1)-\alpha$-D-glucopyranoside. ${ }^{4)}$ Two of the above saccharides, $\beta-\mathrm{F} p 2 \rightarrow 6 \mathrm{G}$ and $\alpha-\mathrm{F} f 2 \rightarrow 6 \mathrm{G}$, can be synthesized from D-glucose and D-fructose using a thermal melting treatment. Several characteristics of these saccharides have been reported. ${ }^{5) 6}$ In this paper, the structure of a novel saccharide, $\beta$-D-fructopyranosyl- $(2 \rightarrow 6)-\beta$ D-fructofuranosyl- $(2 \leftrightarrow 1)-\alpha$-D-glucopyranoside, isolated from the fermented beverage was determined using MALDI-TOF MS and NMR.

†Corresponding author (Tel. +81-134-54-7311, Fax. +81-134-522610, E-mail: oelab-akira@ohtakakohso.co.jp).

Abbreviations: MALDI-TOF MS, matrix-assisted laser desorption ionization time of flight mass spectrometry; HPAEC, high performance anion-exchange chromatography; COSY, correlation spectroscopy; EHSQC, editing heteronuclear single quantum coherence; HSQC-TOCSY, HSQC-total correlation spectroscopy; HMBC, heteronuclear multiple-bond correlation; HR-HMBC, high resolution-HMBC.
Super Ohtaka ${ }^{\circledR}(1 \mathrm{~kg}$, Brix: $59 \%)$ was passed through a carbon-Celite column $(4.5 \times 35 \mathrm{~cm})$ and successively eluted in water $(14 \mathrm{~L})$ and $5 \%$ ethanol $(30 \mathrm{~L})$. Almost all of glucose and fructose were eluted with water $(0-4 \mathrm{~L})$, while the fraction containing the target saccharide named saccharide 1 was eluted in $5 \%$ ethanol fraction $(1-3 \mathrm{~L})$. Then, the fraction containing saccharide $\mathbf{1}$ was concentrated and purified using HPLC systems with an Amide-80 column (Fig. 1) and with an ODS-100V column. Purified saccharide 1 $(1.8 \mathrm{mg})$ was obtained as a white powder. It was determined to be homogeneous based on HPAEC, ${ }^{778) 9}$ where the retention time was $5.51 \mathrm{~min}$ and the relative retention time was 1.10 (the retention time of sucrose is 1.0) (chromatogram not shown). The retention time of saccharide $\mathbf{1}$ did not correspond with any saccharides examined $\left(\mathrm{t}_{\text {Rsucrose }}\right.$; maltose, 1.50; sophorose, 1.26; gentiobiose, 1.59; laminaribiose, 1.33; maltotriose, 2.59; raffinose, 1.23; panose, 1.89; gentiotriose, 1.90; 1-kestose, 1.52; nystose, 2.28). The degree of polymerization (DP) of saccharide 1 was 3 based on measurements of $[\mathrm{M}+\mathrm{Na}]^{+}$ions $(\mathrm{m} / \mathrm{z}: 527)$ using MALDITOF MS. The complete hydrolysis of saccharide 1 was investigated using HPAEC, where saccharide $1(1.0 \mathrm{mg})$ was dissolved in $0.1 \mathrm{~N} \mathrm{HCl}(0.2 \mathrm{~mL})$ and hydrolyzed by heating at $100{ }^{\circ} \mathrm{C}$ for $30 \mathrm{~min}$. As result, glucose and fructose were detected in molar ratio $(0.5: 1.0)$.

NMR analysis for saccharide $\mathbf{1}$ was performed as follows. ${ }^{1} \mathrm{H}$ - and ${ }^{13} \mathrm{C}-\mathrm{NMR}$ signals of saccharide $\mathbf{1}$ were all assigned using 2D-NMR techniques, including COSY, ${ }^{10) 11)} \mathrm{E}-$ HSQC, ${ }^{12) 13)}$ HSQC-TOCSY, ${ }^{12) 14}$ HMBC, ${ }^{15) 16)}$ and HR- 


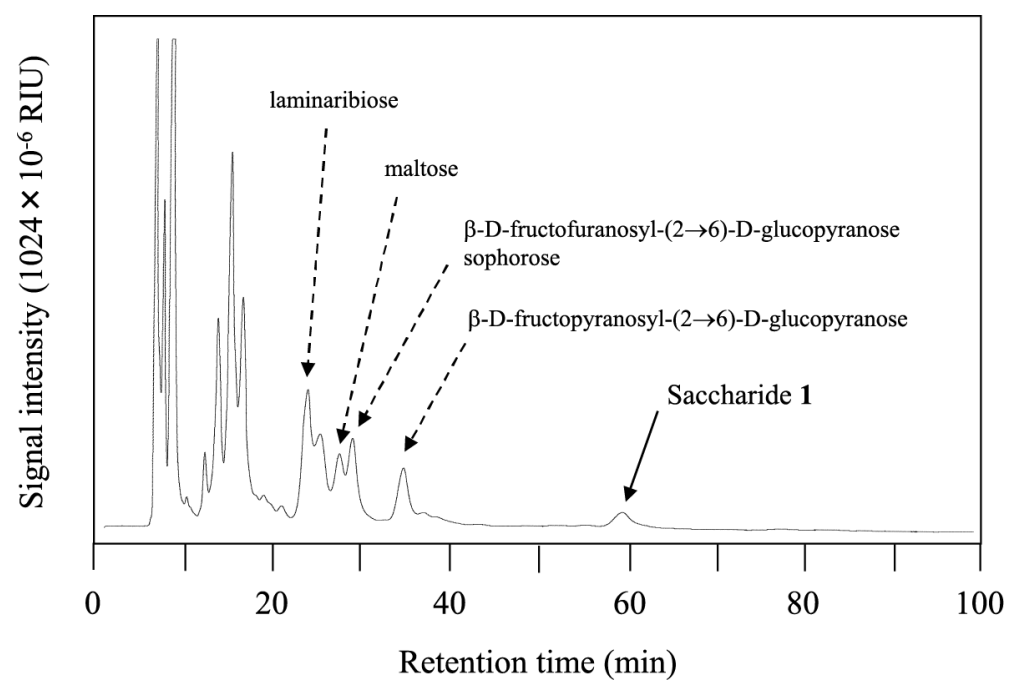

Fig. 1. HPLC of $5 \%$ ethanol fraction separated from fermented beverage of plant extracts by carbon-Celite column chromatography. Fermented beverage of plant extracts was loaded onto a carbon-Celite column and successively eluted with $5 \%$ Ethanol. The following operating conditions for HPLC were used: column, Amide- $80\left(7.8 \times 300 \mathrm{~mm}\right.$; Tosoh Corporation); column temperature, $80{ }^{\circ} \mathrm{C}$; eluent, $80 \%$ acetonitrile; flow rate, $2.0 \mathrm{~mL} / \mathrm{min}$; detection, by refractive index.

HMBC. ${ }^{17)}$ The HSQC-TOCSY spectrum of saccharide 1 revealed ${ }^{1} \mathrm{H}$ and ${ }^{13} \mathrm{C}$ signals of $\mathrm{C}-1(93.05 \mathrm{ppm})$ to $\mathrm{C}-6$ belonging to Glc and signals of C-3 to C-6 belonging to Fru residues. The COSY spectrum assigned the spin system of Glc from H-1 to H-6, Fruf from H-3 to H-6, and Frup from $\mathrm{H}-6$ to $\mathrm{H}-5$. The corresponding ${ }^{13} \mathrm{C}$ signals were assigned from the E-HSQC spectrum. The E-HSQC spectrum gives $\mathrm{CH}_{2}$ signals in opposite phase to $\mathrm{CH}$ and $\mathrm{CH}_{3}$ signals, like DEPT 135. This spectrum of $\mathbf{1}$ discriminated methylene carbons and protons. The HMBC correlations for CFruf-3/H-Fruf-1 and C-Fruf-2/H-Fruf-1 facilitated assignment of H-Fruf-1 and C-Fruf-2. $\beta$-Fructofuranose was assigned based on chemical shifts of $\mathrm{C}-2$ and large value of $J(\mathrm{H}-3 / \mathrm{H}-4)$ and $J(\mathrm{H}-4 / \mathrm{H}-5) .{ }^{18)}$ The HMBC correlations for C-Frup-2/H- Frup-1, C-Frup-2/H-Frup-6, and C-Frup-3/HFru $p-1$ confirmed signal assignment. $\beta$-Fructopyranose was assigned based on the HMBC correlations of C-Frup- $2 / \mathrm{H}$ Frup- 6 and chemical shifts of C-3, 4 , and $5 .{ }^{19)} \mathrm{C}-\mathrm{Fru} f-2\left(\delta_{\mathrm{C}}\right.$ $104.66 \mathrm{ppm}$ ) had an inter-residual HMBC correlation for H-Glc-1 $\left(\delta_{\mathrm{H}} 5.39 \mathrm{ppm}\right)$. The E-HSQC spectrum, shown in Fig. 2A, revealed H-Frup-1 and H-Fruf-6 had similar chemical shifts. Both the intra-residual HMBC correlation peak of C-Frup-2/H-Frup-1 and the inter-residual correlation peak HMBC of C-Frup-2/H-Fruf-6 should appear in the same position in the $2 \mathrm{D}$ spectrum, presented in Fig. $2 \mathrm{~B}$. Discrimination of these two correlations was performed based on the characteristic multiplet pattern of correlation peaks in HR-HMBC spectrum. In the HR-HMBC spectrum, splitting of the cross-peaks due to the long range $J_{\mathrm{CH}}$ and $J_{\mathrm{HH}}$ were scaled by scaling factor $N$ and $N+1$ along the $F_{1}$ dimension, respectively. ${ }^{17)}$ A typical multiplet pattern was shown at the C-Glc-4/H-Glc-3 in the HR-HMBC spectrum of saccharide 1 (Fig. 3). This correlation peak consisted of 6 signals; two sets of three signals aligned in parallel downward-sloping lines. The same pattern was shown at CGlc-2/H-Glc-3. The distance of three signals were corresponding to the proton peak pattern (triplet-like $d d$ ). For the two correlation peaks between C-Fruf-3 and methylene pro- tons of Fruf-1, each signal intensity was weighted by the strong-coupling of methylene protons. Further artificial signals were induced by strong coupling. ${ }^{20}$ ) The correlation peak of C-Frup-3/H-Frup-1 in HR-HMBC spectrum showed as a doublet. That is because two protons of $\mathrm{H}$ Frup-1 had the same chemical shifts indicated in E-HSQC (Fig. 2A), the correlation peak did not split by $J_{\mathrm{HH}}$, therefore, the peak was split only by $N J_{\mathrm{CH}}$. The same doublet pattern was found at C-Frup-2/H-Frup-1. An additional multiplet pattern can be observed at the same position. This multiplet was considered H-Fruf-6 because methylene protons Fruf-6 were the strongly coupled each other (Fig. 2A), they gave the center-weighted multiplet pattern. All ${ }^{1} \mathrm{H}$ - and ${ }^{13} \mathrm{C}$-NMR signals were assigned as shown in Table 1 . Based on these results, saccharide 1 was confirmed to be the novel saccharide $\beta$-D-fructopyranosyl- $(2 \rightarrow 6)-\beta$-D-fructofuranosyl- $(2 \leftrightarrow 1)-\alpha-D-$ glucopyranoside. This saccharide, which is derived from the substitution of the $6^{\mathrm{F}}$ - $\beta$-D-fructopyranosyl residue for the $6^{\mathrm{F}}-\beta$-D-fructofuranosyl residue in 6 -kestose was named pyrano-6-kestose. The purity and yield of saccharide 1 were 98 and $0.18 \%$, respectively.

In this study, the structure of the novel saccharide $\beta$-Dfructopyranosyl- $(2 \rightarrow 6)-\beta$-D-fructofuranosyl- $(2 \leftrightarrow 1)-\alpha-D-$ glucopyranoside was clarified. In addition, this saccharide was confirmed to be produced during fermentation.

\section{EXPERIMENTAL}

Preparation of fermented beverage of plant extracts ( $\mathrm{Su}$ per $\left.\boldsymbol{O h t a k}^{\circledR}\right)$. For preparation of "fermented beverage of plant extracts" (Super Ohtaka ${ }^{\circledR}$; Ohtakakohso Co., Ltd., Otaru, Japan), 50 kinds of fruits and vegetables were used to produce the final extract as shown in previous paper. ${ }^{1)}$ The 50 fruits and vegetable were cut, sliced or diced into small pieces, mixed and put in cedar barrels. Afterwards, equivalent weight of sucrose was added to samples, mixed well to allow high contact samples-sucrose, and then barrels were left during one week at room temperature. The 


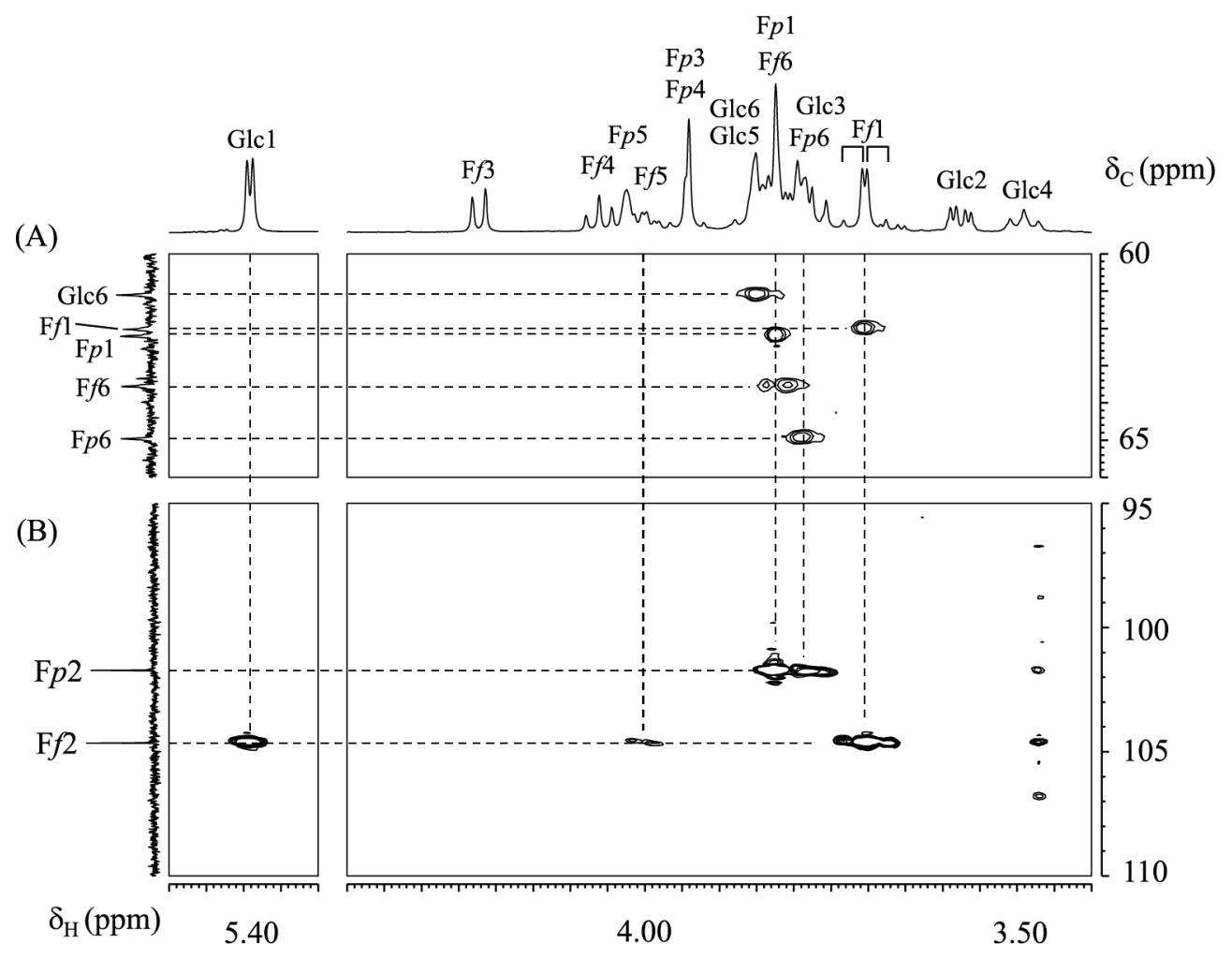

Fig. 2. Portions of E-HSQC (A) and HMBC (B) spectra of saccharide 1.

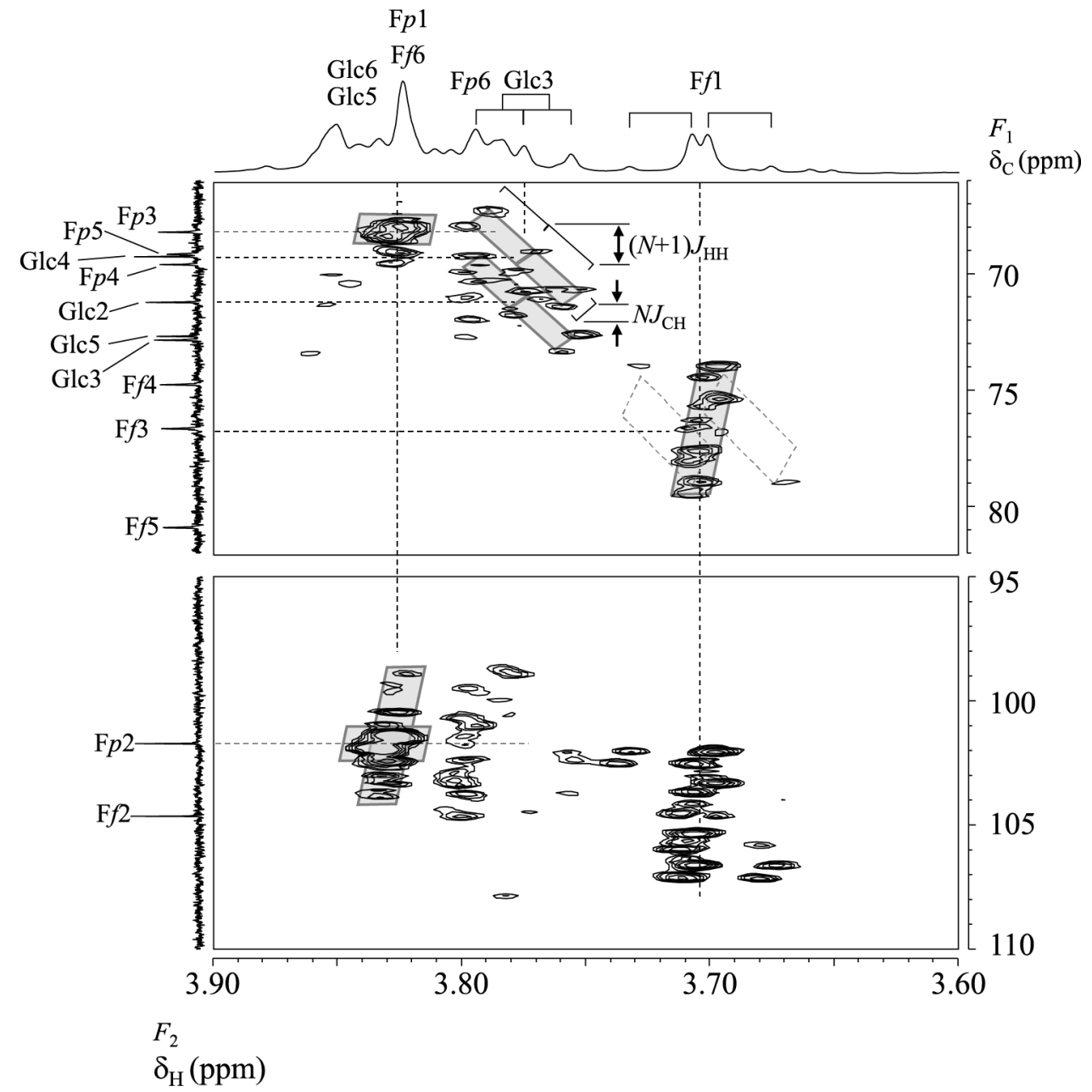

Fig. 3. Portions of HR-HMBC spectrum of saccharide $\mathbf{1 .}$ 
Table 1. ${ }^{1} \mathrm{H}$ and ${ }^{13} \mathrm{C}$ NMR spectral data ( $\delta^{\mathrm{a}}$ in ppm, $J$ in $\mathrm{Hz}$ ) of saccharide 1.

\begin{tabular}{|c|c|c|c|c|c|}
\hline & & $\delta_{\mathrm{C}}$ & $\delta_{\mathrm{H}}$ & & $J_{\mathrm{H}, \mathrm{H}}$ \\
\hline \multirow[t]{7}{*}{ Fru $f$} & 1 & 62.04 & 3.74 & $\mathrm{~d}$ & 13.0 \\
\hline & & & 3.69 & $\mathrm{~d}$ & 13.0 \\
\hline & 2 & 104.66 & & & \\
\hline & 3 & 77.00 & 4.22 & $\mathrm{~d}$ & 8.7 \\
\hline & 4 & 75.23 & 4.06 & $\mathrm{dd}$ & $8.7,8.5$ \\
\hline & 5 & 80.98 & 4.00 & ddd & $8.5,8.0,2.9$ \\
\hline & 6 & 63.57 & 3.81 & $\mathrm{~m}^{\mathrm{b}}$ & \\
\hline \multirow[t]{6}{*}{ Fru $p$} & 1 & 62.22 & 3.82 & $\mathrm{~s}$ & \\
\hline & 2 & 101.74 & & & \\
\hline & 3 & 69.09 & 3.94 & $\mathrm{~m}$ & \\
\hline & 4 & 70.39 & 3.94 & $\mathrm{~m}$ & \\
\hline & 5 & 69.99 & 4.02 & $\mathrm{brs}^{\mathrm{c}}$ & \\
\hline & 6 & 64.97 & 3.79 & $\mathrm{~m}$ & \\
\hline \multirow[t]{6}{*}{ Glc } & 1 & 93.05 & 5.39 & $\mathrm{~d}$ & 3.9 \\
\hline & 2 & 71.91 & 3.58 & $\mathrm{dd}$ & $10.3,3.9$ \\
\hline & 3 & 73.43 & 3.78 & $\mathrm{dd}$ & $10.3,8.7$ \\
\hline & 4 & 70.08 & 3.49 & $\mathrm{dd}$ & $9.5,8.7$ \\
\hline & 5 & 73.28 & 3.84 & $\mathrm{~m}$ & \\
\hline & 6 & 61.12 & 3.85 & $\mathrm{~m}$ & \\
\hline
\end{tabular}

${ }^{\text {a }}$ The chemical shifts of ${ }^{1} \mathrm{H}\left(\delta_{\mathrm{H}}\right)$ and ${ }^{13} \mathrm{C}\left(\delta_{\mathrm{C}}\right)$ in ppm were respectively determined relative to the external standard of sodium $\left[2,2,3,3-{ }^{2} \mathrm{H}_{4}\right]-3$-(trimethylsilyl) propanoate in $\mathrm{D}_{2} \mathrm{O}\left(\delta_{\mathrm{H}} 0.00 \mathrm{ppm}\right)$ and 1,4-dioxane $\left(\delta_{\mathrm{C}} 67.40\right)$ in $\mathrm{D}_{2} \mathrm{O} .{ }^{\mathrm{b}} \mathrm{m}$, multiplet. ${ }^{\mathrm{c}}$ br s, broad singlet. * NMR data of other oligosaccharides containing D-fructofuranosyl or D-fructopyranosyl residue were referred. ${ }^{2122) 23)}$

juice exudates was then separated without compression from solids and used for fermentation. The fermentation beverage was obtained by incubation of the extract at $37^{\circ} \mathrm{C}$ in dark by natural fermentation using yeast (Zygosaccharomyces spp. and Pichia spp.) and lactic acid bacteria (Leuconostoc spp.). After 7 days, the fermented beverage was kept additional maturation and ageing, obtaining finally a brown and slightly sticky liquid.

Isolation of saccharide. Super Ohtaka ${ }^{\circledR}(1 \mathrm{~kg}$, Brix: 59 \%) was loaded onto a $4.5 \times 35 \mathrm{~cm}$ carbon-Celite column consisting of 1:1 charcoal/Celite-535 (Wako Pure Chemical Industries, Ltd., Osaka, Japan) and successively eluted in water $(14 \mathrm{~L})$ and $5 \%$ ethanol $(30 \mathrm{~L})$. Almost all of glucose and fructose were eluted with water $(0-4 \mathrm{~L})$, while the fraction containing the target saccharide named saccharide $\mathbf{1}$ was eluted in $5 \%$ ethanol fraction $(1-3 \mathrm{~L})$. Then, the fraction $(2 \mathrm{~L})$ containing the saccharide was concentrated to 10 $\mathrm{mL}$. Subsequently, the concentrated fraction was repeatedly applied to preparative-HPLC (Tosoh Corporation, Tokyo, Japan) equipped with an Amide-80 column $(7.8 \mathrm{~mm} \times 30$ $\mathrm{cm}$, Tosoh Corporation) at $80{ }^{\circ} \mathrm{C}$ and eluted with $80 \%$ acetonitrile at $2 \mathrm{~mL} / \mathrm{min}$ using refractive index detection (Fig. 1). Furthermore, saccharide 1 was purified at room temperature using an HPLC system (Tosoh Corporation) equipped with an ODS-100V column $(4.6 \mathrm{~mm} \times 25 \mathrm{~cm}$, Tosoh Corporation) and eluted with distilled water at $0.5 \mathrm{~mL} / \mathrm{min}$ using refractive index detection. The purified saccharide $\mathbf{1}$ solution was freeze-dried to give a white powder $(1.8 \mathrm{mg})$.

High performance anion-exchange chromatography (HPAEC). The saccharide was analyzed using Dionex Bio
LC Series (Dionex Corp., Sunnyvale, USA) apparatus equipped with an HPLC carbohydrate column (Carbo Pack PAl, inert styrenedivinylbenzene polymer) and pulsed amperometric detection (PAD). ${ }^{7) 8)}$ The mobile phase ${ }^{9)}$ consisted of eluent A (150 mM NaOH) with eluent B (500 mM sodium acetate in $150 \mathrm{mM} \mathrm{NaOH}$ ) with a sodium acetate gradient as follows: $0-1 \mathrm{~min}, 25 \mathrm{mM}$; $1-2 \mathrm{~min}, 25-50 \mathrm{mM}$; 2-20 min, 50-200 mM; 20-22 min, 500 mM; 22-30 min, $25 \mathrm{mM}$ at a follow rate of $1 \mathrm{~mL} / \mathrm{min}$. The applied PAD potentials for E1 $(500 \mathrm{~ms}), \mathrm{E} 2(100 \mathrm{~ms})$, and E3 (50 ms) were $0.1,0.6$ and $-0.60 \mathrm{~V}$, respectively, and the output range was $1 \mu \mathrm{C}$.

Matrix assisted laser desorption ionization/time of flight mass spectrometry (MALDI-TOF MS). MALDI-TOF MS spectra were measured using a Shimadzu-Kratos mass spectrometer (KOMPACT Prove; Shimadzu Corp., Kyoto, Japan) in positive ion mode with $10 \%$ 2,5-dihydroxylbenzoic acid as the matrix. Ions were formed by a pulsed UV laser beam (nitrogen laser, $337 \mathrm{~nm}$ ). Calibration was conducted using 1-kestose as the external standard.

Nuclear magnetic resonance (NMR) measurements. Saccharide $(1.8 \mathrm{mg})$ was dissolved in $60 \mu \mathrm{L} \mathrm{D}_{2} \mathrm{O}$. NMR spectra were recorded at $27^{\circ} \mathrm{C}$ with a Bruker AMX-500 spectrometer $\left({ }^{1} \mathrm{H} 500 \mathrm{MHz},{ }^{13} \mathrm{C} 126 \mathrm{MHz}\right.$; Bruker BioSpin $\mathrm{GmbH}$, Karlsruhe, Germany) equipped with a $2.5-\mathrm{mm}$ diameter $\mathrm{C} / \mathrm{H}$ dual probe (1D spectra) and a TXI triple probe $(2 \mathrm{D}$ spectra). Chemical shifts in ppm for ${ }^{1} \mathrm{H}\left(\delta_{\mathrm{H}}\right)$ and ${ }^{13} \mathrm{C}\left(\delta_{\mathrm{C}}\right)$ spectra were determined relative to an external standard of sodium $\left[2,2,3,3-{ }^{2} \mathrm{H}_{4}\right]$-3-(trimethylsilyl)-propanoate in $\mathrm{D}_{2} \mathrm{O}\left(\delta_{\mathrm{H}} 0.00 \mathrm{ppm}\right)$ and 1,4 -dioxane $\left(\delta_{\mathrm{C}} 67.40 \mathrm{ppm}\right)$ in $\mathrm{D}_{2} \mathrm{O}$, respectively. COSY, ${ }^{10) 11)}$ E-HSQC, ${ }^{12)}$ HSQC-TOCSY, ${ }^{13) 14)}$ HMBC, ${ }^{15) 16)}$ and HR-HMBC ${ }^{17)}$ spectra were obtained using gradient-selected pulse sequences. The TOCSY mixing time $(0.17 \mathrm{~s})$ was determined using the decoupling in the presence of scalar interactions (DIPSI)-2 methods.

Full NMR spectra are provided in Supplementary material.

\section{REFERENCES}

1) H. Okada, K. Kudoh, E. Fukushi, S. Onodera, J. Kawabata, and N. Shiomi: Antioxidative activity and protective effect of fermented plant extract on ethanol-induced damage to rat gastric mucosa. J. Jpn. Soc. Nutr. Food Sci., 58, 209215 (2005).

2) H. Okada, E. Fukushi, A. Yamamori, N. Kawazoe, S. Onodera, J. Kawabata, and N. Shiomi: Isolation and structural confirmation of the oligosaccharides containing $\alpha$-D-fructofuranoside linkage isolated from fermented beverage of plant extracts. Carbohydr. Res., 346, 2633-2637 (2011).

3) N. Kawazoe, H. Okada, E. Fukushi, A. Yamamori, S. Onodera, J. Kawabata, and N. Shiomi: Two novel saccharides isolated from fermented beverage of plant extract. Carbohydr. Res., 343, 549-554 (2008).

4) H. Okada, E. Fukushi, A. Yamamori, N. Kawazoe, S. Onodera, J. Kawabata, and N. Shiomi: Novel fructopyranose oligosaccharides isolated from fermented beverage of plant extract. Carbohydr. Res., 345, 414-418 (2010). 
5) A. Yamamori, H. Okada, N. Kawazoe, S. Onodera, and N. Shiomi: Synthesis of $\beta$-D-fructopyranosyl-(2 $\rightarrow 6)$-D-glucopyranose from D-glucose and D-fructose by thermal treatment. Biosci. Biotechnol. Biochem., 74, 2130-2132 (2010).

6) A. Yamamori, H. Okada, N. Kawazoe, K. Muramatsu, S. Onodera, and N. Shiomi: Characteristics of $\alpha$-D-fructofuranosyl-( $\rightarrow 6)$-D-glucopyranose synthesized from D-glucose and D-fructose by thermal treatment. J. Appl. Glycosci., 61, 99-104 (2014).

7) R.D. Rocklin and C.A. Pohl: Determination of carbohydrate by anion exchange chromatography with pulse amperometric detection. J. Liq. Chromatogr., 6, 1577-1590 (1983).

8) D.C. Johnson: Carbohydrate detection gains potential. $\mathrm{Na}$ ture, 321, 451-452 (1986).

9) N. Shiomi, S. Onodera, N.J. Chatterton, and P.A. Harrison: Separation of fructooligosaccharide isomer by anion-exchange chromatography. Agric. Biol. Chem., 55, 14271428 (1991).

10) W.P. Aue, E. Batrholdi, and R.R. Ernst: Two-dimensional spectroscopy. Application to nuclear magnetic resonance. J. Chem. Phys., 64, 2229-2246 (1976).

11) M. von Kienlin, C.T.W Moonen, A. von der Toorn, and P.C.M. van Zijl: Rapid recording of solvent-supressed 2D COSY spectra with inherent quadrature detection using pulsed fieldgradients. J. Magn. Reson., 93, 423-429 (1991).

12) W. Willker, D. Leibfritz, R. Kerssebaum, and W. Bermel: Gradient selection in inverse heteronuclear correlation spectroscopy. Magn. Reson. Chem., 31, 287-292 (1993).

13) A. Yamamori, E. Fukushi, S. Onodera, J. Kawabata, and N. Shiomi: NMR analysis of mono- and difructosyllactosucrose synthesized by $1^{\mathrm{F}}$-fructosyltransferase purified from roots of asparagus (Asparagus officinalis L.). J. Magn. Reson., 40, 541-544 (2002).

14) T. Domke: A new method to distinguish between direct and remote signals in proton-relayed $\mathrm{X}, \mathrm{H}$ correlations. $J$.
Magn. Reson., 95, 174-177 (1991).

15) A. Bax and M.F. Summers: ${ }^{1} \mathrm{H}$ and ${ }^{13} \mathrm{C}$ assignments from sensitivity-enhanced detection of heteronuclear multiplebond connectivity by 2D multiple quantum NMR. J. Am. Chem. Soc., 108, 2093-2094 (1986).

16) R.E. Hurd and B.K. John: Gradient-enhanced protondetected heteronuclear multiple-quantum coherence spectroscopy. J. Magn. Reson., 91, 648-653 (1991).

17) K. Furihata, M. Tashiro, and H. Seto: High resolutionHMBC (HR-HMBC), a new method for measuring heteronuclear long-range coupling constants. Magn. Reson. Chem., 48, 179-183 (2010).

18) D. Doddrell and A. Allerhand: Study of anomeric equilibra of ketose in water by natural abundanece carbon-13 Fourie transform nuclear magnetic resonance. D-fructose and Dturanose. J. Am. Chem. Soc., 93, 2779 (1971).

19) H.B. Sinclair: Two methyl anhydro-D-fructopyranosides prepared from D-mannitol. Carbohydr. Res., 181, 115-123 (1988).

20) P. Würtz, P. Permi, N.C. Nielsen, and O.W. Sørensen: Clean HMBC: Suppression of strong-coupling induced artifacts in HMBC spectra. J. Magn. Reson., 194, 89-98 (2008).

21) H. Okada, N. Kawazoe, A. Yamamori, S. Onodera, and N. Shiomi: Structural analysis and characteristics of oligosaccharides isolated from fermented beverage of plant extract. Dyn. Biochem. Process Biotechnol. Mol. Biol., 1, 1-9 (2009).

22) N. Shiomi, T. Abe, H. Kikuchi, Y. Takata, E. Fukushi, Y. Fukushi, J. Kawabata, K. Ueno, and S. Onodera: Structural analysis of novel kestose isomers isolated from sugar beet molasses. Carbohydr. Res., 424, 1-7 (2016).

23) A. Yamamori, Y. Takata, E. Fukushi, J. Kawabata, H. Okada, N. Kawazoe, K. Ueno, S. Onodera, and N. Shiomi: Structural analysis of novel low-digestible sucrose isomers synthesized from D-glucose and D-fructose by thermal treatment. J. Appl. Glycosci., 64, 15-19 (2017). 